\title{
Physical activity and quality of life in patients with pulmonary hypertension
}

\begin{abstract}
To the Editor:
Pulmonary arterial hypertension (PAH) is a progressive, non-curable disease with significant associated comorbidity, including reduced exercise capacity [1] and poor health-related quality of life (HRQoL) [2]. Exercise capacity is frequently measured using the 6-min walk test (6MWT) [3]. The 6MWT is easy to perform and correlates with disease severity and HRQoL [4]. However, the 6MWT has limitations such as the need for a clinic visit to perform it [4] and ceiling effects which reduce the sensitivity to changes among patients [5].
\end{abstract}

Commercially available activity trackers have the potential to monitor ambulatory function continuously beyond one-time measures of exercise capacity. For the PAH population, data on ambulatory activity may provide a more sensitive means to assess changes in clinical status [4] and be informative in the assessment of new therapies. However, an understanding of the long-term correlation between physical activity and both 6-min walking distance (6MWD) and patient-centred outcomes, such as HRQoL, is needed as prior studies were limited to short-term follow-up [6-9].

The primary aims of this prospective cohort study were to assess the correlation between 6MWD and physical activity (step counts) and to examine associations between physical activity and HRQoL among patients with PAH and chronic thromboembolic pulmonary hypertension (CTEPH) over an extended period.

We enrolled patients $\geqslant 18$ years-old from the University of Michigan Medical Center between June and November 2016 at the time of a PAH clinic visit and followed them until their next scheduled appointment. Participants were included if they had a diagnosis of PAH or CTEPH according to World Health Organization (WHO) criteria [10] and WHO functional class I to III symptoms [3] on stable PAH medications for $\geqslant 3$ months without comorbid conditions that limited activity. The study was approved by the Institutional Review Board and informed consent was obtained (HUM00110649).

At each visit, a 6MWT was performed and physical activity quantified using average daily step counts for 2 weeks acquired with the Fitbit Zip accelerometer (Fitbit, San Francisco, CA, USA). Days with $<200$ steps were excluded and weeks with $\geqslant 4$ excluded days were considered non-valid. Participants were instructed to wear the Fitbit Zip during waking hours and to continue their usual level of activity. HRQoL was measured using the emPHasis-10 questionnaire, a PAH-specific instrument used to determine the impact of PAH symptoms on patients' lives [11], and several non-disease specific Patient-Reported Outcomes Measurement Information System (PROMIS) short-form domain measures (physical function-v1.2, fatigue-v1.0, illness impact-v1.0, satisfaction with roles-v1.0 and ability to participate in social roles-v2.0) [12].

The primary outcomes of the study were: 1) correlation of 6MWT and physical activity, 2) association between physical activity and HRQoL, and 3) association between physical activity change and HRQoL change between baseline and follow-up. Visual inspection of scatterplots suggested a curvilinear relationship between step counts and 6MWD. The inclusion of step count as a second-order term (quadratic) improved the model fit. A p-value of $<0.05$ was used to determine significance. Analyses were conducted using STATA 14 (StataCorp LP, College Station, TX, USA).

Of the 87 consecutive patients screened, 36 either didn't meet inclusion criteria or met exclusion criteria, 11 were eligible but did not participate, and one enrolled but elected not to participate, leaving 39

@ERSpublications

In a longitudinal study of stable pulmonary hypertension patients, there was a nonlinear association between 6MWD and step counts at higher levels of exercise capacity and average step counts were associated with disease-specific and global HRQoL http://bit.ly/2JiCvRX

Cite this article as: Cascino TM, McLaughlin VV, Richardson CR, et al. Physical activity and quality of life in patients with pulmonary hypertension. Eur Respir J 2019; 53: 1900028 [https://doi.org/10.1183/ 13993003.00028-2019]. 
participants in the study (mean \pm SD age $61 \pm 12$ years, $85 \%(n=33)$ female, $90 \%$ PAH $(n=35)$ and $10 \%$ CTEPH $(n=4))$. A total of 34 participants $(87 \%)$ had valid mean step counts for the initial visit, $69 \%$ $(n=27)$ for the follow-up visit, and $64 \%(n=25)$ for both and were included in the change between visits analysis. Participants were followed for a median (interquartile range) of 6.1 (4.6-6.4) months between visits.

The mean \pm SD steps and 6 MWD were $4391 \pm 2442$ steps and $460 \pm 107 \mathrm{~m}$. Average daily step count was significantly associated with $6 \mathrm{MWD}\left(\mathrm{R}=0.71, \mathrm{R}^{2}=0.51, \mathrm{p}<0.001\right)$ (figure $\left.1 \mathrm{a}\right)$. Average daily step counts and HRQoL measures remained stable between visits. Higher average daily step counts were positively associated with lower pulmonary hypertension symptoms burden (emPHasis-10 score) and PROMIS domains, including higher self-reported function, lower fatigue, higher satisfaction with social roles and decreased psychosocial illness impact (figure 1b). The adjusted analyses examining the association between change in step counts and change in HRQoL measures are also reported in figure $1 \mathrm{~b}$ and reflect the clinical stability in all measures between visits.

Our findings support the stability and reliability of continuous long-term monitoring of physical activity in PAH and CTEPH patients. Over a median follow-up of about 6 months in clinically stable participants, physical activity measured by average daily step counts did not change and was strongly associated with 6MWD. The current use of 6MWD to monitor exercise capacity is well-established. Thus, 6MWD is frequently used as an efficacy outcome in clinical trials [13]. However, given the limitations, there has been increasing interest in using continuous device monitoring to assess ambulatory function [4]. The confirmation of the stability and reliability of physical activity using measures over an extended period builds on prior work of shorter duration and is a necessary first step in understanding if physical activity monitoring adequately addresses these limitations [6-9].

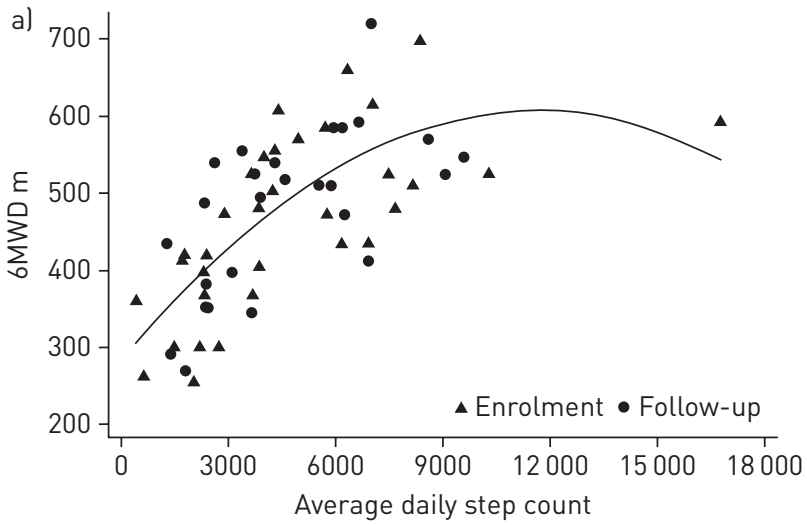

b)

\begin{tabular}{|c|c|c|c|c|c|c|}
\hline & \multirow[b]{2}{*}{ emPHasis-10 } & \multicolumn{5}{|c|}{ PROMIS domains } \\
\hline & & $\begin{array}{l}\text { Physical } \\
\text { function }\end{array}$ & $\begin{array}{l}\text { Participate in } \\
\text { social roles }\end{array}$ & Fatigue & $\begin{array}{l}\text { Psychosocial } \\
\text { illness impact }\end{array}$ & $\begin{array}{l}\text { Satisfaction with } \\
\text { social roles }\end{array}$ \\
\hline \multicolumn{7}{|c|}{ Baseline and follow-up } \\
\hline $\begin{array}{l}\text { Step count (per } \\
1000 \text { steps) }\end{array}$ & $-1.690(0.463)$ & $1.425(0.373)$ & $0.826(0.538)$ & $-1.534(0.521)$ & $-1.020(0.398)$ & $1.419(0.520)$ \\
\hline $\mathrm{p}$-value & 0.001 & 0.001 & 0.133 & 0.006 & 0.015 & 0.010 \\
\hline $\mathrm{R}^{2}$ & 0.24 & 0.32 & 0.10 & 0.19 & 0.17 & 0.19 \\
\hline \multicolumn{7}{|c|}{ Change between visits } \\
\hline $\begin{array}{l}\text { Step count (per } \\
1000 \text { steps) }\end{array}$ & $0.008(0.534)$ & $-0.107(0.256)$ & $-0.465(0.543)$ & $0.859(0.692)$ & $1.361(0.744)$ & $-0.058(0.717)$ \\
\hline$p$-value & $>0.20$ & $>0.20$ & $>0.20$ & $>0.20$ & 0.081 & $>0.20$ \\
\hline $\mathrm{R}^{2}$ & 0.05 & 0.44 & 0.15 & 0.13 & 0.16 & 0.04 \\
\hline
\end{tabular}

FIGURE 1 a) Scatter plot with regression line showing the relationship between 6-min walking distance (6MWD) and average daily step counts. Average daily step count was significantly associated with 6MWD $\left(R=0.63, R^{2}=0.39, p<0.001\right)$. Model fit improved when step counts were included as a second order variable $\left(R=0.71, R^{2}=0.51, p<0.001\right)$ (shown in figure). b) Results of the multivariable linear regressions adjusting for age and sex. Health-related quality of life (HRQoL) and change in $H R Q \circ L$ were the dependent variables with average daily step counts and change in average daily step counts the primary independent variables. Regression coefficient with standard errors in parentheses, $\mathrm{p}$-value and $\mathrm{R}^{2}$ displayed for each model. The emPHasis-10 score ranges from 0-50 with higher scores representing worse symptom burden and quality of life [12]. Patient-Reported Outcomes Measurement Information System (PROMIS) results are transformed using a T-score metric with a mean of 50 and a standard deviation of 10 for the general US population. A higher score indicates more of the domain being evaluated [13]. 
In addition, there was a high correlation between physical activity and $6 \mathrm{MWD}$, which has been previously described [6-8]. However, we observed this relationship to be nonlinear suggesting that at higher ambulatory activity (daily step counts), the 6MWD lost discriminatory value. This may represent the previously reported ceiling effect where the $6 \mathrm{MWT}$ was unable to differentiate response to therapies in participants with higher exercise capacity [5]. With the use of early combination therapies, participants enrolling in clinical trials are changing and tend to have improved exercise tolerance limiting the ability to use 6MWD in these patients [14]. Our findings suggest that ambulatory step count monitoring has the potential to be more sensitive to changes in functional capacity among active individuals.

Ambulatory step count monitoring in our study was associated with HRQoL, including the emPHasis-10 survey and several general health domains. These findings are particularly important given the evolving emphasis at the World Symposium on Pulmonary Hypertension on translating clinical trial results into meaningful outcomes for participants rather than less patient-centred outcomes such as 6MWD [15]. As a result, home activity monitoring has the potential to offer a more complete understanding of ambulatory activity and should be considered as an exploratory patient-centred outcome in clinical trials.

Our study has limitations. Overall, participants found using the Fitbit Zip to be acceptable, wearing the device for $77 \%$ of eligible days. Despite this, $36 \%$ of participants (14 out of 39) did not provide step count data at both visits. Involving patients in the research design may better elucidate the optimal strategy to improve compliance. Although our median follow-up was 6 months, the duration was too short in this clinically stable group to detect changes in physical activity and HRQoL. A larger, longer study or a study aimed at increasing physical activity is necessary to determine if physical activity is sensitive to change or if improving physical activity improves clinically important outcomes.

Our results suggest step counts provide a clinically useful method for measuring ambulatory function among patients with PAH and CTEPH, which correlated with 6MWD and HRQoL. It is recommended that investigators using devices which measure step counts examine their data for nonlinear correlations with other measures. Finally, given the relationship of daily physical activity and HRQoL, future studies which promote regular physical activity with a goal of improving HRQoL are warranted.

Thomas M. Cascino', Vallerie V. McLaughlin', Caroline R. Richardson ${ }^{2}$, Nilofar Behbahani-Nejad ${ }^{3}$, Victor M. Moles ${ }^{1}$, Scott H. Visovatti ${ }^{1}$ and Elizabeth A. Jackson ${ }^{4}$

${ }^{1}$ Division of Cardiovascular Medicine, University of Michigan, Ann Arbor, MI, USA. ${ }^{2}$ Dept of Family Medicine, University of Michigan, Ann Arbor, MI, USA. ${ }^{3}$ Oakland University William Beaumont School of Medicine, Auburn Hills, MI, USA. ${ }^{4}$ Division of Cardiovascular Medicine, University of Alabama at Birmingham, Birmingham, AL, USA.

Correspondence: Thomas M. Cascino, 2381 CVC SPC 5853, 1500 E. Medical Center Drive, Ann Arbor, MI 48109-5853, USA. E-mail: tcascino@med.umich.edu

Received: Dec 182018 | Accepted after revision: March 062019

Conflict of interest: T.M. Cascino reports grants from National Institutes of Health and from University of Michigan Frankel Cardiovascular Center, during the conduct of the study. V.V. McLaughlin reports personal fees from Actelion Pharmaceuticals US, Inc., Bayer, Gilead Sciences, Inc., Medtronic, Merck, St. Jude Medical and United Therapeutics Corporation, and grants from Actelion Pharmaceuticals US, Inc., Arena, Bayer and Sonovie (University of Michigan received research funding), all outside the submitted work. C.R. Richardson has nothing to disclose. N. Behbahani-Nejad has nothing to disclose. V.M. Moles has nothing to disclose. S.H. Visovatti has nothing to disclose. E.A. Jackson has nothing to disclose.

Support statement: This study was funded by a Michigan Medicine Frankel Cardiovascular Center Micro Grant and National Institutes of Health T32 postdoctoral research training grant (T32-HL007853). Funding information for this article has been deposited with the Crossref Funder Registry.

\section{References}

1 Delcroix M, Howard L. Pulmonary arterial hypertension: the burden of disease and impact on quality of life. Eur Respir Rev 2015; 24: 621-629.

2 Mathai SC, Suber T, Khair RM, et al. Health-related quality of life and survival in pulmonary arterial hypertension. Ann Am Thorac Soc 2016; 13: 31-39.

3 Galie N, Humbert M, Vachiery JL, et al. 2015 ESC/ERS Guidelines for the diagnosis and treatment of pulmonary hypertension: The Joint Task Force for the Diagnosis and Treatment of Pulmonary Hypertension of the European Society of Cardiology (ESC) and the European Respiratory Society (ERS): Endorsed by: Association for European Paediatric and Congenital Cardiology (AEPC), International Society for Heart and Lung Transplantation (ISHLT). Eur Heart J 2016; 37: 67-119.

4 McLaughlin V, Bacchetta M, Badesch D, et al. Update on pulmonary arterial hypertension research: proceedings from a meeting of experts. Curr Med Res Opin 2018; 34: 263-273.

5 Frost AE, Langleben D, Oudiz R, et al. The 6-min walk test (6MW) as an efficacy endpoint in pulmonary arterial hypertension clinical trials: demonstration of a ceiling effect. Vascul Pharmacol 2005; 43: 36-39.

6 Mainguy V, Provencher S, Maltais F, et al. Assessment of daily life physical activities in pulmonary arterial hypertension. PLoS One 2011; 6: e27993. 
7 Pugh ME, Buchowski MS, Robbins IM, et al. Physical activity limitation as measured by accelerometry in pulmonary arterial hypertension. Chest 2012; 142: 1391-1398.

8 Matura LA, Shou H, Fritz JS, et al. Physical activity and symptoms in pulmonary arterial hypertension. Chest 2016; 150: 46-56.

9 Okumus G, Aslan GK, Arseven O, et al. The role of an activity monitor in the objective evaluation of patients with pulmonary hypertension. Clin Respir J 2018; 12: 119-125.

10 McLaughlin VV, Archer SL, Badesch DB, et al. ACCF/AHA 2009 expert consensus document on pulmonary hypertension a report of the American College of Cardiology Foundation Task Force on Expert Consensus Documents and the American Heart Association developed in collaboration with the American College of Chest Physicians; American Thoracic Society, Inc.; and the Pulmonary Hypertension Association. J Am Coll Cardiol 2009; 53: 1573-1619.

11 Yorke J, Corris P, Gaine S, et al. emPHasis-10: development of a health-related quality of life measure in pulmonary hypertension. Eur Respir J 2014; 43: 1106-1113.

12 Cella D, Yount S, Rothrock N, et al. The Patient-Reported Outcomes Measurement Information System (PROMIS): progress of an NIH Roadmap cooperative group during its first two years. Med Care 2007; 45: Suppl. 1, S3-S11.

13 Demir R, Kucukoglu MS. Six-minute walk test in pulmonary arterial hypertension. Anatol J Cardiol 2015; 15: 249-254.

14 McLaughlin VV. Has the 6-min walk distance run its course? Chest 2012; 142: 1363-1365.

15 McGoon MD, Ferrari P, Armstrong I, et al. The importance of patient perspectives in pulmonary hypertension. Eur Respir J 2019; 53: 1801919. 